\begin{abstract}
Discussion
Comparing the intramuscular ergometrine and syntometrine in the management of the third stage of labour in this study, there is a reduction in the mean length of the third stage from 10.5 to 7.8 minutes and in the total blood loss from 8.3 to $6 \mathrm{oz}$. (235 to $170 \mathrm{ml}$.). There are two cases of post-partum haemorrhage in the syntometrine group, an incidence of $2 \%$; excluding the case due to vaginal laceration, the incidence is $1 \%$. For the ergometrine group there are 11 cases of post-partum haemorrhage, an incidence of 7\%. In 1960 the overall incidence of post-partum haemorrhage at St. Mary's Hospitals was $5 \%$; in 1954 it was $6.8 \%$.

Statistical calculations show that the reduction in the length of the third stage of labour and the incidence of retained placenta may be due to chance. There is more significance in the reduction of total blood loss and incidence of post-partum haemorrhage, though these results still remain within the realms of chance.
\end{abstract}

\section{Conclusion and Summary}

Syntometrine is proved to be equally as satisfactory and shows promise of being better than intramuscular ergometrine in the management of the third stage of labour. It has the added advantage of containing both syntocinon 5 I.U. and ergometrine $0.5 \mathrm{mg}$. in the same ampoule in stable combination.

I am grateful to Professor W. I. C. Morris for his advice and help, also to the labour ward sisters for their continued co-operation throughout this trial. The syntometrine used was supplied by Sandoz Products Ltd., through the courtesy of Dr. N. Holgate.

REFERENCE

Embrey, M. P. (1961). Brit. med. J., 1, 1737.

\section{Medical Memoranda}

\section{Rupture of Uterus Following Shirodkar Suture}

The following case is reported merely to emphasize the possible dangers of this procedure, which have been mentioned by Meoldy (1958), Page (1958), Shirodkar (1960), Fluhmann (1961), and Isaacs and Pavlic (1962).

\section{CASE History}

In 1958 the patient, then aged 32, was admitted to hospital as an emergency case of threatened abortion. She was about 17 weeks in her first pregnancy. On examination the cervix was dilated four fingerbreadths, with the feet of the foetus protruding through the vulva. Abortion occurred almost immediately after admission.

In 1959 she was readmitted when 13 weeks pregnant, and as the cervix was already starting to be taken up, this was reinforced with an $18-\mathrm{lb}$. (8.2-kg.) tensile-strength braided nylon suture. No complications occurred after this procedure, and a week later she was permitted to go home. Three months later she was again admitted to the ward, draining blood-stained liquor. Severe painful contractions began, and the nylon suture was removed. She subsequently aborted; the duration of her pregnancy on this occasion was 24 weeks.
In October, 1961, she was seen again, when 11 weeks pregnant, and in view of her previous history it was again decided to adopt the Shirodkar technique. This time a No. 5 " ethicon" ligature was used. No complications occurred and she was discharged home after one week. Two months later, when her pregnancy had advanced to 21 weeks, she was readmitted with slight vaginal bleeding and some uterine contractions. These continued in spite of heavy sedation, and the foetus was aborted six hours after admission. As there was only slight bleeding after the abortionwhich occurred at midnight - it was decided not to take the patient to the theatre for evacuation until the next morning. She continued to lose slightly during the night.

Examination in the theatre revealed a vertical tear in the posterior surface of the uterus. The tear extended from above the nylon ligature in the lower segment up to the fundus of the uterus, for a distance of about 3 in. $(7.5 \mathrm{~cm}$.). The uterine laceration communicated with the vagina, through which the foetus had been expelled, and extended up into the peritoneal cavity. After an episiotomy to improve the approach the uterine tear was with some difficulty sutured via the vaginal route by means of interrupted mattress No. 1 chromic-catgut sutures.

\section{COMMENT}

In this case the anxiety of the staff and the patient for her third pregnancy to continue to viability was naturally very high. The danger of a ruptured uterus, if uterine contractions were allowed to go on too long with a suture in the cervix, was not fully appreciated. It is suggested, therefore, that if contractions are strong the suture must be removed before serious damage occurs to the uterus, even though one knows that this will result in the patient aborting and that her own and her attendants' hopes of a viable baby will be shattered.

In view of this patient's history of uterine irritability, her present age (36 years), and the severe uterine damage, it was reluctantly decided to advise against further pregnancies.

\section{J. G. Thurston, F.R.C.O.G..}

Consultant Obstetrician and Gynaecologist, Blackburn Group of Hospitals.

REFERENCES Fluhmann, C. F. (1961). The Cervix Uteri and its Diseases.
Isaacs, J. H., and Pavlic, R. S. (1962). Amer. J. Obstet. Gynec., Isaacs, J. H., and Pavlic, R. S. (1962). Amer. J. Obstet. Gynec., Meoldy, G. F. (1958). Quoted by R. B. Durfee, Obstet. and Gynec., 1958, 12, 91 .

Page, E. W. (1958). Obstet. and Gynec., 12, 509.

Page, E. W. (1958). (1960). Contributions to Obstetrics and Gynaecology, ist ed., p. 3 .

In his annual report for the year 1961, Dr. James Hutton, the medical officer of health for the Borough of Poole, Dorset, states that the final stage of the diversion of all sewage from its present discharge into Poole Bay to the new sewage disposal works at Broadstone has now been entered into. Poole contains some 10 miles of bathing beaches, and at present 8 outfalls discharge sewage into Bournemouth and Poole Bays. It is hoped that the final diversion of sewage away from the bay in Poole for treatment of sewage disposal works will be completed within the next few years. Only four cases of pollution of the harbour occurred during 1961 ; three are stated to have been of a minor character, and the fourth was the result of a collision between two ships. Examinations of shellfish from Poole Bay under the Public Health (Shell Fish) Regulations, 1934 and 1948, showed that out of 120 samples none were contaminated by faecal coli to the extent of Class III (unsatisfactory), and only one sample could be described as Class II (suspicious). 